$\angle 8$ Research Square

\title{
Frailty in Patients Undergoing Surgery for Brain Tumors: A Systematic Review of the Literature
}

\author{
Sakibul Huq \\ University of Pittsburgh Medical Center \\ Jiaqi Liu \\ Johns Hopkins University School of Medicine \\ Robert Romano \\ Johns Hopkins University School of Medicine \\ Stella Seal \\ Johns Hopkins University School of Medicine \\ Adham Khalafallah \\ University of Miami Miller School of Medicine: University of Miami School of Medicine \\ Jeremy D. Walston \\ Johns Hopkins University School of Medicine \\ Debraj Mukherjee ( $\nabla$ dmukher1@jhmi.edu) \\ Johns Hopkins University School of Medicine https://orcid.org/0000-0002-5403-8237
}

\section{Research Article}

Keywords: frailty, brain tumor, oncology, outcomes

Posted Date: February 25th, 2022

DOI: https://doi.org/10.21203/rs.3.rs-1382210/v1

License: (c) (i) This work is licensed under a Creative Commons Attribution 4.0 International License. Read Full License 


\section{Abstract}

\section{Introduction}

Emerging literature suggests that frailty may be an important driver of postoperative outcomes in patients undergoing surgery for brain tumors. We systematically reviewed the literature on frailty in brain tumor patients with respect to three questions: What methods of frailty assessment have been applied to brain tumor patients? What thresholds have been defined to distinguish between different levels of frailty? What clinical outcomes does frailty predict in brain tumor patients?

\section{Methods}

A literature search was conducted using PubMed, Embase, The Cochrane Library, Web of Science, Scopus, and ClinicalTrials.gov. Included studies were specific to brain tumor patients, used a validated instrument to assess frailty, and measured the impact of frailty upon postoperative outcomes.

\section{Results}

Of 753 citations, 21 studies met our inclusion criteria. Frailty instruments studied, in order of frequency reported, included the 5-factor modified frailty index, 11-factor modified frailty index, Johns Hopkins Adjusted Clinical Groups frailty-defining diagnosis indicator, and Hopkins Frailty Score. Multiple different conventions and thresholds for distinguishing levels of frailty were reported. Clinical outcomes associated with frailty included mortality, survival, complications, length of stay (LOS), charges, costs, discharge disposition, readmissions, and operative time.

\section{Conclusions}

Frailty is an increasingly popular concept in brain tumor patients that is associated with important clinical outcomes. However, the extant literature is largelycomprised of retrospective studies with heterogeneous definitions of frailty, thresholds for defining levels of frailty, and patient populations. Further work is needed to understand best practices in assessing frailty inbrain tumor patients and applying these concepts to clinical practice.

\section{Introduction}

Frailty is a state of decreased reserves and resistance to stressors that is associated with adverse health outcomes in older adults[1]. Many conceptual models of frailty have been proposed over the past three decades[2]. Early theories focused on loss of adaptability and vulnerability to stressors leading to disability and disease[3]. In 2001, two popular models emerged. Rockwood described frailty as an accumulation of age-related deficits across multiple domains of health that cumulatively lead to poor outcomes[4]. In contrast, Fried described a frailty phenotype based on a decline in physiologic reserves from underlying aging processes, resulting in decreased capacity to respond to stress[1,2].

In 2010, Makary and colleagues published a landmark paper extending Fried's frailty phenotype to surgical patients[5]. Related studies since then have consistently demonstrated that this phenotype, often called physical frailty, is an important driver of postoperative outcomes. Recently, several index-type tools that aggregate multiple co-morbid medical conditions common in older adults have emerged. These include the Modified Frailty Index [including 11-factor (mFI-11)[6] and 5-factor (mFI-5)[7] versions] and the Johns Hopkins Adjusted Clinical Groups (JHACG) frailty-defining diagnosis indicator[8]among others. In these cases, the term frailty is utilized as a potential marker of vulnerability to adverse outcomes. There is growing consensus that frailty measurement, no matter what the conceptual etiological framework, should help identify a vulnerable subset of older adults and facilitate shared decision-making with patients regarding incumbent risk of surgery[9,10].

More recently, frailty measurement has been explored in neurosurgery, with emerging literature suggesting that it may be a salient concept in surgical brain tumor patients[11-31]. Frailty represents a potentially modifiable characteristic in this patient population with significant implications for clinical decision-making, prediction of postoperative outcomes, and high-value care[18]. However, this nascent literature is largely comprised of retrospective studies with significant heterogeneity in frailty assessment methodology[32].

To inform future research and clinical applications of different types of frailty measurement in neurosurgical oncology, we systematically reviewed the extant literature on frailty in surgical brain tumor patients. To focus our review, we sought to answer three questions:

1. What methods of frailty measurement have been applied to brain tumor patients?

2. What thresholds have been defined to distinguish between different levels and types of frailty?

3. What clinical outcomes do the various categories of frailty measurement predict in brain tumor patients?

\section{Methods}

\section{Electronic Literature Search}


A systematic review of the English peer-reviewed literature was conducted by a librarian (SS) following Preferred Reporting Items for Systematic Reviews and Meta-Analyses (PRISMA) guidelines (Fig. 1)[33]. PubMed, Embase, Cochrane Library, Web of Science, Scopus, and Clinicaltrials.gov were queried for all reports related to frailty in patients undergoing surgery for brain tumors between January 1, 1950 and April 6, 2021 . The search employed three concepts: "brain tumor pathology," "undergoing surgery," and "frailty" (Supplementary Table 1).

\section{Study Eligibility}

Included studies were specific to brain tumor patients, used a quantitative instrument to assess frailty, and described the impact of frailty upon postoperative outcomes. Only fully published, peer-reviewed studies in the primary medical literature were included. Studies were excluded if they did not include brain tumor patients, did not measure frailty, did not report postoperative outcomes, included fewer than two patients or results in nonhuman models, or were not in English.

\section{Data Extraction}

Title and abstract screening was performed on all eligible studies by two independent reviewers (RR, JL) using Covidence Systematic Review Software (Melbourne, Australia) based on a priori inclusion and exclusion criteria. A third reviewer (SH) evaluated decisions and resolved disagreements. Articles passing title and abstract screening underwent full-text review by three reviewers (SH, RR, JL).

\section{Synthesis and Assessment of Literature}

Included studies were qualitatively synthesized, with a focus on comparisons between methods of frailty assessment, thresholds defining different levels of frailty, and outcomes are shown to be influenced by frailty. Level of evidence was assessed for all studies using Oxford Center for EvidenceBased Medicine (OCEBM) guidelines[34]. Study quality was assessed using the Newcastle-Ottawa Scale, which judges studies based on the selection of study groups, comparability of groups, and ascertainment of the outcome of interest[35]. Each included study was evaluated and assigned a total score ranging from zero to nine. Studies with five or more points were considered high quality[36, 37].

\section{Results}

\section{Search Results and Assessment of Evidence}

Our search identified 753 citations (Fig. 1). After 278 duplicates were removed by Covidence, 475 records underwent title and abstract screening, resulting in 111 studies eligible for full-text review. Of these, 12 studies were duplicates, 1 was not available online, 29 were abstract-only, 15 were not primary medical literature, 18 did not report or clearly define preoperative frailty, 5 were not specific to brain tumor patients or did not distinguish brain tumor patients from other included patients, and 10 did not report an association between frailty and postoperative outcomes.

A total of 21 studies were included in our analysis. All studies were published between 2016 and 2021 . A total of 13 studies analyzed singleinstitution databases, while eight studies analyzed data from national registries (one National Inpatient Sample, three Nationwide Readmissions Database, four National Surgical Quality Improvement Program (NSQIP)). A total of 20 studies were retrospective and considered level IV evidence according to OCEBM guidelines (Table 1). One study (Harland et al., 2020) was prospective and considered level Ilb evidence. All studies were considered high quality according to the Newcastle-Ottawa Scale (Table 2). However, we note that all retrospective studies may be subject to selection, publication, and reporting biases and may therefore be limited in their generalizability. 
Table 1

Assessment of available literature. Level of evidence determined using Oxford Center for Evidence-Based Medicine guidelines.

\begin{tabular}{|ll|}
\hline Authors \& Year & Level of Evidence \\
\hline Asemota et al., 2019 & IV \\
\hline Bonney et al., 2021 & IV \\
\hline Casazza et al., 2020 & IV \\
\hline Cloney et al., 2016 & IV \\
\hline Goshtasbi et al., 2020 & IV \\
\hline Harland et al., 2020 & IIb \\
\hline Henry et al., 2021 & IV \\
\hline Huq et al., 2020 & IV \\
\hline Huq et al., 2021 & IV \\
\hline Jimenez et al., 2020 & IV \\
\hline Katiyar et al., 2020 & IV \\
\hline Khalafallah et al., 2020 & IV \\
\hline Khalafallah et al., 2020 & IV \\
\hline Khalafallah et al., 2020 & IV \\
\hline Richardson et al., 2019 & IV \\
\hline Sastry et al., 2020 & IV \\
\hline Schneider et al., 2020 & IV \\
\hline Shahrestani et al., 2020 & IV \\
\hline Shahrestani et al., 2020 & IV \\
\hline Theriault et al., 2020 & IV \\
\hline Youngerman et al., 2018 & IV \\
\hline
\end{tabular}


Table 2

Study quality assessment using Newcastle-Ottawa scale for non-randomized studies.

\begin{tabular}{|c|c|c|c|c|c|c|c|c|c|}
\hline \multirow{2}{*}{$\begin{array}{l}\text { Authors \& } \\
\text { Year }\end{array}$} & \multicolumn{4}{|l|}{ Selection } & \multirow{2}{*}{$\begin{array}{l}\text { Comparability } \\
\text { Comparability } \\
\text { of cohorts on } \\
\text { the basis of } \\
\text { design or } \\
\text { analysis }\end{array}$} & \multicolumn{3}{|l|}{ Outcome } & \multirow{2}{*}{$\begin{array}{l}\text { Total } \\
\text { score }\end{array}$} \\
\hline & $\begin{array}{l}\text { Representative } \\
\text { ness of the } \\
\text { exposed cohort }\end{array}$ & $\begin{array}{l}\text { Selection } \\
\text { of non- } \\
\text { exposed } \\
\text { cohort }\end{array}$ & $\begin{array}{l}\text { Ascertain- } \\
\text { ment of } \\
\text { exposure }\end{array}$ & $\begin{array}{l}\text { Demonstration } \\
\text { that outcome } \\
\text { was not } \\
\text { present at } \\
\text { start of study }\end{array}$ & & $\begin{array}{l}\text { Assessment } \\
\text { of outcome }\end{array}$ & $\begin{array}{l}\text { Follow-up } \\
\text { long } \\
\text { enough } \\
\text { for } \\
\text { outcomes } \\
\text { to occur }\end{array}$ & $\begin{array}{l}\text { Adequacy } \\
\text { of follow } \\
\text { up }\end{array}$ & \\
\hline $\begin{array}{l}\text { Asemota et } \\
\text { al., } 2019\end{array}$ & 1 & 1 & 1 & 0 & 2 & 1 & 0 & 1 & 7 \\
\hline $\begin{array}{l}\text { Bonney et } \\
\text { al., } 2021\end{array}$ & 1 & 1 & 1 & 0 & 2 & 1 & 1 & 1 & 8 \\
\hline $\begin{array}{l}\text { Casazza et } \\
\text { al., } 2020\end{array}$ & 1 & 1 & 1 & 0 & 2 & 1 & 1 & 1 & 8 \\
\hline $\begin{array}{l}\text { Cloney et al., } \\
2016\end{array}$ & 0 & 1 & 1 & 0 & 2 & 1 & 1 & 1 & 7 \\
\hline $\begin{array}{l}\text { Goshtasbi et } \\
\text { al., } 2020\end{array}$ & 1 & 1 & 1 & 0 & 0 & 1 & 1 & 1 & 6 \\
\hline $\begin{array}{l}\text { Harland et } \\
\text { al., } 2020\end{array}$ & 1 & 1 & 1 & 0 & 2 & 1 & 0 & 1 & 7 \\
\hline $\begin{array}{l}\text { Henry et al., } \\
2021\end{array}$ & 1 & 1 & 1 & 0 & 2 & 1 & 0 & 1 & 7 \\
\hline $\begin{array}{l}\text { Huq et al., } \\
2020\end{array}$ & 1 & 1 & 1 & 0 & 2 & 1 & 0 & 1 & 7 \\
\hline $\begin{array}{l}\text { Huq et al., } \\
2021\end{array}$ & 1 & 1 & 1 & 1 & 2 & 1 & 1 & 1 & 9 \\
\hline $\begin{array}{l}\text { Jimenez et } \\
\text { al., } 2020\end{array}$ & 1 & 1 & 1 & 1 & 2 & 1 & 1 & 1 & 9 \\
\hline $\begin{array}{l}\text { Katiyar et } \\
\text { al., } 2020\end{array}$ & 1 & 1 & 1 & 0 & 2 & 1 & 1 & 1 & 8 \\
\hline $\begin{array}{l}\text { Khalafallah } \\
\text { et al., } 2020\end{array}$ & 1 & 1 & 1 & 1 & 2 & 1 & 1 & 1 & 9 \\
\hline $\begin{array}{l}\text { Khalafallah } \\
\text { et al., } 2020\end{array}$ & 1 & 1 & 1 & 1 & 2 & 1 & 1 & 1 & 9 \\
\hline $\begin{array}{l}\text { Khalafallah } \\
\text { et al., } 2020\end{array}$ & 1 & 1 & 1 & 1 & 2 & 1 & 1 & 1 & 9 \\
\hline $\begin{array}{l}\text { Richardson } \\
\text { et al., } 2019\end{array}$ & 1 & 1 & 1 & 1 & 2 & 1 & 1 & 1 & 9 \\
\hline $\begin{array}{l}\text { Sastry et al., } \\
2020\end{array}$ & 1 & 1 & 1 & 0 & 2 & 1 & 0 & 1 & 7 \\
\hline $\begin{array}{l}\text { Schneider et } \\
\text { al, } 2020\end{array}$ & 0 & 1 & 1 & 0 & 2 & 1 & 0 & 1 & 6 \\
\hline $\begin{array}{l}\text { Shahrestani } \\
\text { et al., } 2020\end{array}$ & 1 & 1 & 1 & 0 & 2 & 1 & 1 & 1 & 8 \\
\hline $\begin{array}{l}\text { Shahrestani } \\
\text { et al., } 2020\end{array}$ & 1 & 1 & 1 & 0 & 2 & 1 & 1 & 1 & 8 \\
\hline $\begin{array}{l}\text { Theriault et } \\
\text { al., } 2020\end{array}$ & 0 & 1 & 1 & 1 & 2 & 1 & 1 & 1 & 8 \\
\hline $\begin{array}{l}\text { Youngerman } \\
\text { et al., } 2018\end{array}$ & 1 & 1 & 1 & 0 & 2 & 1 & 1 & 1 & 8 \\
\hline
\end{tabular}

\section{Frailty Instruments Studied}

Four frailty instruments were reported (Table 3). The most common (reported in nine studies) was the mFI-5, comprised of five variables: hypertension, diabetes, chronic obstructive pulmonary disease, congestive heart failure, and functional status[7]. 
Table 3

Frailty instruments reported in included studies. Third column indicates thresholds used to delineate different levels of frailty in each study. References provided below.

\begin{tabular}{|c|c|c|}
\hline Frailty Instrument & Components & Thresholds/Cutpoints \\
\hline \multirow[t]{5}{*}{5 -factor modified frailty index ( $\mathrm{mFI}-5)$} & • Hypertension & - Non-frail (0) vs. low frailty (1) vs. moderate-to-high frailty $(\geq 2)[1-5]$ \\
\hline & - Diabetes & - Non-frail (0) vs. low frailty (0.2) vs. medium-to-high frailty $(>0.2)[6]$ \\
\hline & $\cdot$ COPD & - Dichotomized at the median: 0 vs. $\geq 1$ [7] \\
\hline & $\cdot \mathrm{CHF}$ & - Used as continuous variable[8, 9$]$ \\
\hline & - Functional status & \\
\hline \multirow{8}{*}{$\begin{array}{l}\text { 11-factor modified frailty index (mFl- } \\
\text { 11) }\end{array}$} & mFI-5 plus: & - Low frailty $(0)$ vs. moderate frailty $(1-2)$ vs. high frailty $(\geq 3)[10,11]$ \\
\hline & $\cdot \mathrm{Ml}$ & - Robust (0) vs. non-frail (0 or 1) vs. prefrail (1) vs. frail ( $\geq 2$ ) [12] \\
\hline & • Prior PCl/angina & $\begin{array}{l}\text { - None }(0) \text { vs. low frailty }(0.01-0.19) \text { vs. intermediate frailty }(0.2-0.29) \text { vs. } \\
\text { high frailty }(\geq 0.3)[13]\end{array}$ \\
\hline & $\begin{array}{l}\text { - PVD/ischemic rest } \\
\text { pain }\end{array}$ & - Least-frail $(0-0.08)$ vs. moderately-frail $(0.09-0.26)$ vs. frailest $(\geq 0.27)[14]$ \\
\hline & - Impaired & - Dichotomized at the median: 0 vs. $\geq 1[7]$ \\
\hline & sensorium & $\cdot$ Good medical health $(<2)$ vs. $\geq 2[15]$ \\
\hline & $\cdot$ CVA/TIA & - Non-frail (0) vs. frail $(\geq 1)[16]$ \\
\hline & - CVA with deficit & \\
\hline \multirow{10}{*}{$\begin{array}{l}\text { Johns Hopkins } \\
\text { Adjusted Clinical Groups (JHACG) } \\
\text { frailty-defining } \\
\text { diagnosis indicator }\end{array}$} & - Malnutrition & - Binary scale: 0 vs. $\geq 1$ [17-20] \\
\hline & - Dementia & \\
\hline & $\begin{array}{l}\text { - Severe vision } \\
\text { impairment }\end{array}$ & \\
\hline & - Decubitus ulcer & \\
\hline & $\begin{array}{l}\text { - Urinary } \\
\text { incontinence }\end{array}$ & \\
\hline & - Fecal incontinence & \\
\hline & -Weight loss & \\
\hline & • Difficulty walking & \\
\hline & $\cdot$ Falls & \\
\hline & $\begin{array}{l}\text { - Social support } \\
\text { need }\end{array}$ & \\
\hline \multirow[t]{5}{*}{ Hopkins Frailty Score (HFS) } & • Shrinking & - Non-frail $(0-1)$ vs. moderately frail $(2-3)$ vs. frail $(4-5)$ [21] \\
\hline & -Weakness & \\
\hline & • Exhaustion & \\
\hline & - Low activity & \\
\hline & $\begin{array}{l}\text { - Slowed walking } \\
\text { speed }\end{array}$ & \\
\hline
\end{tabular}

Abbreviations: CHF: congestive heart failure. COPD: chronic obstructive pulmonary disease. MI: myocardial infarction. PCI: percutaneous coronary intervention. PVD: peripheral vascular disease. TIA: transient ischemic attack

1. Goshtasbi K, Abouzari M, Soltanzadeh-Zarandi S, Sarna B, Lee A, Hsu FPK, Djalilian HR (2020) The association of age, body mass index, and frailty with vestibular schwannoma surgical morbidity. Clinical Neurology and Neurosurgery 197 doi:10.1016/j.clineuro.2020.106192

2. Henry RK, Reeves RA, Wackym PA, Ahmed OH, Hanft SJ, Kwong KM (2021) Frailty as a Predictor of Postoperative Complications Following Skull Base Surgery. The Laryngoscope doi:10.1002/lary.29485

10.1002/lary.29485.

3. Huq S, Khalafallah AM, Jimenez AE, Gami A, Lam S, Ruiz-Cardozo MA, Oliveira LAP, Mukherjee D (2020) Predicting Postoperative Outcomes in Brain Tumor Patients With a 5-Factor Modified Frailty Index. Neurosurgery doi:10.1093/neuros/nyaa335

4. Huq S, Khalafallah AM, Patel P, Sharma P, Dux H, White T, Jimenez AE, Mukherjee D (2021) Predictive Model and Online Calculator for Discharge Disposition in Brain Tumor Patients. World neurosurgery 146: e786-e798 doi:10.1016/j.wneu.2020.11.018 
5. Jimenez AE, Khalafallah AM, Huq S, Horowitz MA, Azmeh O, Lam S, Oliveira LAP, Brem H, Mukherjee D (2020) Predictors of Nonroutine Discharge Disposition Among Patients with Parasagittal/Parafalcine Meningioma. World neurosurgery 142: e344-e349 doi:10.1016/j.wneu.2020.06.239

10.1016/j.wneu.2020.06.239. Epub 2020 Jul 9.

6. Sastry RA, Pertsch NJ, Tang 0, Shao B, Toms SA, Weil RJ (2020) Frailty and outcomes after craniotomy for brain tumor. J Clin Neurosci 81 : 95-100 doi:10.1016/j.jocn.2020.09.002

10.1016/j.jocn.2020.09.002. Epub 2020 Oct 2.

7. Khalafallah AM, Huq S, Jimenez AE, Brem H, Mukherjee D (2020) The 5-factor modified frailty index: an effective predictor of mortality in brain tumor patients. Journal of neurosurgery doi:10.3171/2020.5.Jns20766

8. Khalafallah AM, Shah PP, Huq S, Jimenez AE, Patel PP, London NR, Hamrahian AH, Salvatori R, Gallia GL, Rowan NR, Mukherjee D (2020) The 5-factor modified frailty index predicts health burden following surgery for pituitary adenomas. Pituitary doi:10.1007/s11102-020-01069-5

9. Khalafallah AM, Jimenez AE, Patel P, Huq S, Azmeh O, Mukherjee D (2020) A novel online calculator predicting short-term postoperative outcomes in patients with metastatic brain tumors. Journal of neuro-oncology doi:10.1007/s11060-020-03626-1

10. Cloney M, D'Amico R, Lebovic J, Nazarian M, Zacharia BE, Sisti MB, Bruce JN, McKhann GM, Iwamoto FM, Sonabend AM (2016) Frailty in Geriatric Glioblastoma Patients: A Predictor of Operative Morbidity and Outcome. World neurosurgery 89: 362-367

doi:10.1016/j.wneu.2015.12.096

11. Katiyar V, Sharma R, Tandon V, Goda R, Ganeshkumar A, Suri A, Chandra PS, Kale SS (2020) Impact of frailty on surgery for glioblastoma: a critical evaluation of patient outcomes and caregivers' perceptions in a developing country. Neurosurgical focus 49: E14 doi:10.3171/2020.7.Focus20482

10.3171/2020.7.FOCUS20482.

12. Casazza GC, McIntyre MK, Gurgel RK, McCrary HC, Shelton C, Couldwell WT, Bowers CA (2020) Increasing Frailty, Not Increasing Age, Results in Increased Length of Stay Following Vestibular Schwannoma Surgery. Otol Neurotol 41: E1243-E1249 doi:10.1097/mao.0000000000002831

13. Youngerman BE, Neugut Al, Yang J, Hershman DL, Wright JD, Bruce JN (2018) The modified frailty index and 30-day adverse events in oncologic neurosurgery. Journal of neuro-oncology 136: 197-206 doi:10.1007/s11060-017-2644-0

14. Schneider M, Potthoff AL, Scharnböck E, Heimann M, Schäfer N, Weller J, Schaub C, Jacobs AH, Güresir E, Herrlinger U, Vatter H, Schuss P (2020) Newly diagnosed glioblastoma in geriatric $(65+)$ patients: impact of patients frailty, comorbidity burden and obesity on overall survival. Journal of neuro-oncology 149: 421-427 doi:10.1007/s11060-020-03625-2

10.1007/s11060-020-03625-2. Epub 2020 Sep 29.

15. Richardson AM, McCarthy DJ, Sandhu J, Mayrand R, Guerrero C, Rosenberg C, Gernsback JE, Komotar R, Ivan M (2019) Predictors of Successful Discharge of Patients on Postoperative Day 1 After Craniotomy for Brain Tumor. World neurosurgery 126: e869-e877 doi:10.1016/j.wneu.2019.03.004

16. Theriault BC, Pazniokas J, Adkoli AS, Cho EK, Rao N, Schmidt M, Cole C, Gandhi C, Couldwell WT, Al-Mufti F, Bowers CA (2020) Frailty predicts worse outcomes after intracranial meningioma surgery irrespective of existing prognostic factors. Neurosurgical focus 49 : E16 doi:10.3171/2020.7.Focus20324

10.3171/2020.7.FOCUS20324.

17. Asemota AO, Gallia GL (2019) Impact of frailty on short-term outcomes in patients undergoing transsphenoidal pituitary surgery. Journal of neurosurgery 132: 360-370 doi:10.3171/2018.8.Jns181875

10.3171/2018.8.JNS181875.

18. Shahrestani S, Ballatori AM, Chen XT, Ton A, Strickland BA, Brunswick A, Zada G (2020) Analysis of modifiable and nonmodifiable risk factors in patients undergoing pituitary surgery. Journal of neurosurgery: 1-8 doi:10.3171/2020.4.Jns20417

10.3171/2020.4.JNS20417.

19. Shahrestani S, Lehrich BM, Tafreshi AR, Brown NJ, Lien BV, Ransom S, Ransom RC, Ballatori AM, Ton A, Chen XT, Sahyouni R (2020) The Role of Frailty in Geriatric Cranial Neurosurgery for Primary Central Nervous System Neoplasms. Neurosurgical focus 49: 1-8 doi:10.3171/2020.7.FOCUS20426

20. Bonney PA, Chartrain AG, Briggs RG, Jarvis CA, Ding L, Mack WJ, Zada G, Attenello FA (2021) Frailty Is Associated with In-Hospital Morbidity and Nonroutine Disposition in Brain Tumor Patients Undergoing Craniotomy. World neurosurgery 146: e1045-e1053 doi:10.1016/j.wneu.2020.11.083

21. Harland TA, Wang M, Gunaydin D, Fringuello A, Freeman J, Hosokawa PW, Ormond DR (2020) Frailty as a Predictor of Neurosurgical Outcomes in Brain Tumor Patients. World neurosurgery 133: e813-e818 doi:10.1016/j.wneu.2019.10.010

Most studies using the $\mathrm{mFI}-5$ categorized patients into three levels: non-frail, low frailty, or medium/moderate-to-high frailty. Studies by Goshtasbi[15], Henry[17], Huq[18, 19], and Jimenez[20] delineated these levels based on mFl-5 scores of 0, 1, or $\geq 2$, respectively. These integer values represent the number of positive elements on the $\mathrm{mFI}-5$ scale. Sastry used corresponding fractions, with the number of present frailty 
elements divided by five [26]. Two studies by Khalafallah did not delineate different frailty categories but used the mFI-5 as a continuous variable; a third study dichotomized the $\mathrm{mFI}-5$ at the median (0 vs. $\geq 1)[21-23]$.

The second most-reported index (in eight studies) was the $\mathrm{mFI-11}$, which includes the $\mathrm{mFI}-5$ plus myocardial infarction, prior percutaneous coronary intervention, peripheral vascular disease/ischemic rest pain, impaired sensorium, cerebrovascular accident/transient ischemic attack, and cerebrovascular accident with deficit[18]. Reported thresholds in $\mathrm{mFI}-11$ scores differed by study and were similarly reported as either integers or as fractions. Cloney and Katiyar categorized patients into three integer-based categories: low frailty (0) vs. moderate frailty (1-2) vs. high frailty ( $\geq 3$ ) $[14,24]$. Schneider used three similar categories reported as fractions based on a denominator of 11 (least-frail (0-0.08) vs. moderately-frail (0.090.26 ) vs. frailest $(\geq 0.27)$ )[27]. Casazza used four categories: robust (0) vs. non-frail (0 or 1$)$ vs. prefrail (1) vs. frail ( $\geq 2)$ [13]. Youngerman used four categories reported as fractions (none (0) vs. low frailty $(0.01-0.19)$ vs. intermediate frailty $(0.2-0.29)$ vs. high frailty ( $\geq 0.3)$ ), with the denominator representing the number of variables recorded for that patient in the NSQIP database [31]. This denominator was less than 11 in the case of missing data. Khalafallah dichotomized $\mathrm{mFI}-11$ at the median of 1[22]. Two categories of frailty were also used in studies by Theriault ( 0 vs. $\geq 1)$ [30] and Richardson (<2 vs. $\geq 2)[25]$.

Four studies used the JHACG frailty-defining diagnosis indicator, in which patients were designated as frail or non-frail based on having 0 or $\geq 1$ of the following diagnoses: malnutrition, dementia, severe vision impairment, decubitus ulcer, urinary incontinence, fecal incontinence, weight loss, difficulty walking, falls, or social support needs[8, 11, 12, 28, 29].

Harland and colleagues used the HFS, which includes shrinking, weakness, exhaustion, low activity, and slowed walking speed[1]. Patients were designated as non-frail (0-1), moderately frail (2-3), or frail (4-5)[16].

\section{Clinical Outcomes}

Clinical outcomes reportedly associated with each frailty instrument are described in Table 4, with additional detail in Supplementary Table 2. Frailty status as ascertained by the $\mathrm{mFI}-5$ was associated with mortality, complications, LOS, charges, discharge disposition, operation time, and readmissions[15, 17-20, 22, 26]. Frailty status using the mFI-11 was associated with in-hospital mortality, overall survival, complications, LOS, charges, discharge disposition, end of life care needs, and reoperation rates[13, 14, 22, 24, 25, 27, 30, 31]. Frailty using the JHACG tool was associated with mortality, complications, reoperation rates, LOS, charges, costs, discharge disposition, and readmissions[11, 12, 28, 29]. Frailty as measured by the HFS tool was associated with complications, discharge disposition, and LOS[16]. 
Table 4

Studies included in systematic review.

\begin{tabular}{|c|c|c|c|c|c|}
\hline $\begin{array}{l}\text { Authors \& } \\
\text { Year }\end{array}$ & Study Type & Data Source & $\begin{array}{l}\text { Frailty } \\
\text { instrument }\end{array}$ & Study population & Outcomes Studied \\
\hline $\begin{array}{l}\text { Asemota et } \\
\text { al., } 2019\end{array}$ & Retrospective & $\begin{array}{l}\text { National } \\
\text { Inpatient Sample } \\
\text { Database }\end{array}$ & JHACG & $\begin{array}{l}\text { •Patients who underwent transsphenoidal } \\
\text { surgery for pituitary tumors/disorders } \\
\cdot n=115,317\end{array}$ & $\begin{array}{l}\cdot \text { Mortality rates } \\
\cdot \text { Complications } \\
\cdot \text { Reoperation rates } \\
\cdot \text { LOS } \\
\cdot \text { Discharge disposition } \\
\cdot \text { Hospitalization costs }\end{array}$ \\
\hline $\begin{array}{l}\text { Bonney et } \\
\text { al., } 2021\end{array}$ & Retrospective & $\begin{array}{l}\text { Nationwide } \\
\text { Readmissions } \\
\text { Database }\end{array}$ & JHACG & $\begin{array}{l}\text { Patients who underwent craniotomy for } \\
\text { brain tumors, except pituitary tumors or } \\
\text { biopsy } \\
\cdot n=87,835\end{array}$ & $\begin{array}{l}\text {-Complications } \\
\text {-Discharge disposition } \\
\text {-Readmissions (30d and } \\
\text { 90d) }\end{array}$ \\
\hline $\begin{array}{l}\text { Casazza et } \\
\text { al., } 2020\end{array}$ & Retrospective & $\begin{array}{l}\text { Single institution } \\
\text { database }\end{array}$ & $\mathrm{mFI}-11$ & $\begin{array}{l}\cdot \text { Patients who underwent surgery for } \\
\text { vestibular schwannoma } \\
\cdot n=218\end{array}$ & $\begin{array}{l}\cdot \text { Complications } \\
\cdot \text { LOS }\end{array}$ \\
\hline $\begin{array}{l}\text { Cloney et al., } \\
2016\end{array}$ & Retrospective & $\begin{array}{l}\text { Single institution } \\
\text { database }\end{array}$ & $\mathrm{mFI}-11$ & $\begin{array}{l}\cdot \text { Patients }>65 \text { years who underwent } \\
\text { craniotomy for glioblastoma } \\
\cdot n=243\end{array}$ & $\begin{array}{l}\cdot \text { Overall survival } \\
\cdot \text { Complications } \\
\cdot \text { LOS } \\
\cdot \text { Total hospital charges }\end{array}$ \\
\hline $\begin{array}{l}\text { Goshtasbi et } \\
\text { al., } 2020\end{array}$ & Retrospective & NSQIP database & $\mathrm{mFl}-5$ & $\begin{array}{l}\cdot \text { Patients who underwent surgery for } \\
\text { vestibular schwannoma } \\
\cdot n=1,405\end{array}$ & $\begin{array}{l}\cdot \text { Complications } \\
\cdot \text { LOS } \\
\text {-Operation time }\end{array}$ \\
\hline $\begin{array}{l}\text { Harland et } \\
\text { al., } 2020\end{array}$ & Prospective & $\begin{array}{l}\text { Single institution } \\
\text { database }\end{array}$ & HFS & $\begin{array}{l}\cdot \text { Patients who underwent surgery for brain } \\
\text { tumors } \\
\cdot n=260\end{array}$ & $\begin{array}{l}\cdot \text { Complications } \\
\cdot \text { LOS } \\
\cdot \text { Discharge disposition }\end{array}$ \\
\hline $\begin{array}{l}\text { Henry et al., } \\
2021\end{array}$ & Retrospective & NSQIP database & $\mathrm{mFI}-5$ & $\begin{array}{l}\cdot \text { Patients who underwent skull base } \\
\text { procedures } \\
\cdot n=17,912\end{array}$ & $\begin{array}{l}\text {-Overall complications } \\
\text {-Life-threatening (Clavien- } \\
\text { Dindo grade IV) } \\
\text { complications } \\
\text {-Mortality } \\
\text {-LOS }\end{array}$ \\
\hline $\begin{array}{l}\text { Huq et al., } \\
2020\end{array}$ & Retrospective & $\begin{array}{l}\text { Single institution } \\
\text { database }\end{array}$ & $\mathrm{mFI}-5$ & $\begin{array}{l}\text { Patients who underwent surgery for brain } \\
\text { tumors } \\
\cdot n=1,692\end{array}$ & $\begin{array}{l}\cdot \text { Complications } \\
\text { •LOS } \\
\cdot \text { Total hospital charges }\end{array}$ \\
\hline $\begin{array}{l}\text { Huq et al., } \\
2021\end{array}$ & Retrospective & $\begin{array}{l}\text { Single institution } \\
\text { database }\end{array}$ & $\mathrm{mFI}-5$ & $\begin{array}{l}\cdot \text { Patients who underwent surgery for brain } \\
\text { tumors } \\
\cdot n=2,335\end{array}$ & -Discharge disposition \\
\hline $\begin{array}{l}\text { Jimenez et } \\
\text { al., } 2020\end{array}$ & Retrospective & $\begin{array}{l}\text { Single institution } \\
\text { database }\end{array}$ & $\mathrm{mFI}-5$ & $\begin{array}{l}\cdot \text { Patients who underwent surgery for } \\
\text { parasagittal/parafalcine meningioma } \\
\cdot n=154\end{array}$ & -Discharge disposition \\
\hline
\end{tabular}

Abbreviations: LOS: length of stay. NSQIP: National Surgical Quality Improvement Program. 


\begin{tabular}{|c|c|c|c|c|c|}
\hline $\begin{array}{l}\text { Authors \& } \\
\text { Year }\end{array}$ & Study Type & Data Source & $\begin{array}{l}\text { Frailty } \\
\text { instrument }\end{array}$ & Study population & Outcomes Studied \\
\hline \multirow[t]{5}{*}{$\begin{array}{l}\text { Katiyar et } \\
\text { al., } 2020\end{array}$} & Retrospective & $\begin{array}{l}\text { Single institution } \\
\text { database }\end{array}$ & $\mathrm{mFl}-11$ & $\begin{array}{l}\text {-Patients who underwent biopsy or resection } \\
\text { of glioblastoma } \cdot n=276 \text { ( } 93 \text { elderly) }\end{array}$ & $\cdot$ In-hospital mortality \\
\hline & & & & & •Overall survival \\
\hline & & & & & •Complications \\
\hline & & & & & $\cdot$ LOS \\
\hline & & & & & •End of life care needs \\
\hline \multirow{3}{*}{$\begin{array}{l}\text { Khalafallah } \\
\text { et al., } 2020\end{array}$} & Retrospective & Single institution & $\mathrm{mFI}-5$ & -Patients who underwent endoscopic & $\cdot$ LOS \\
\hline & & & & 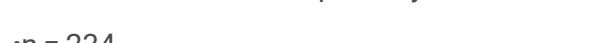 & •Total charges \\
\hline & & & & & •Readmissions \\
\hline \multirow[t]{2}{*}{$\begin{array}{l}\text { Khalafallah } \\
\text { et al., } 2020\end{array}$} & Retrospective & $\begin{array}{l}\text { Single institution } \\
\text { database }\end{array}$ & $\begin{array}{l}\mathrm{mFI}-5, \\
\mathrm{mFI}-11\end{array}$ & $\begin{array}{l}\text {-Patients who underwent surgery for brain } \\
\text { tumors }\end{array}$ & •90-day mortality \\
\hline & & & & $\cdot n=1,692$ & \\
\hline \multirow{3}{*}{$\begin{array}{l}\text { Khalafallah } \\
\text { et al., } 2020\end{array}$} & Retrospective & Single institution & $\mathrm{mFI}-5$ & -Patients who underwent surgery for & •Extended LOS (> 7 days) \\
\hline & & & & & -Discharge disposition \\
\hline & & & & & $\begin{array}{l}\text { •High hospital charges } \\
(>\$ 46,083)\end{array}$ \\
\hline \multirow[t]{2}{*}{$\begin{array}{l}\text { Richardson } \\
\text { et al., } 2019\end{array}$} & Retrospective & $\begin{array}{l}\text { Single institution } \\
\text { database }\end{array}$ & $\mathrm{mFl}-11$ & $\begin{array}{l}\text {-Patients who underwent craniotomy for } \\
\text { brain tumors }\end{array}$ & $\begin{array}{l}\text {-Discharge on } \\
\text { postoperative day } 1\end{array}$ \\
\hline & & & & $\cdot n=424$ & \\
\hline \multirow{5}{*}{$\begin{array}{l}\text { Sastry et al., } \\
2020\end{array}$} & Retrospective & NSQIP database & $\mathrm{mFI}-5$ & $\begin{array}{l}\text {-Patients who underwent craniotomy for } \\
\text { brain tumors }\end{array}$ & -Major complications \\
\hline & & & & (1) & -Discharge disposition \\
\hline & & & & & ·30-day readmissions \\
\hline & & & & & ·30-day mortality \\
\hline & & & & $\cdot n=20,333$ & \\
\hline \multirow[t]{2}{*}{$\begin{array}{l}\text { Schneider et } \\
\text { al., } 2020\end{array}$} & Retrospective & $\begin{array}{l}\text { Single institution } \\
\text { database }\end{array}$ & $\mathrm{mFl}-11$ & $\begin{array}{l}\text {-Patients who underwent surgery for } \\
\text { glioblastoma }\end{array}$ & •1-year mortality \\
\hline & & & & $\cdot n=110$ & • Overall survival \\
\hline \multirow[t]{3}{*}{$\begin{array}{l}\text { Shahrestani } \\
\text { et al., } 2020\end{array}$} & Retrospective & $\begin{array}{l}\text { Nationwide } \\
\text { Readmissions }\end{array}$ & JHACG & $\begin{array}{l}\text {-Geriatric patients who underwent surgery for } \\
\text { primary CNS neoplasms }\end{array}$ & •Complications \\
\hline & & Database & & & $\cdot$ LOS \\
\hline & & & & & -Costs \\
\hline \multirow{4}{*}{$\begin{array}{l}\text { Shahrestani } \\
\text { et al., } 2020\end{array}$} & Retrospective & $\begin{array}{l}\text { Nationwide } \\
\text { Readmissions }\end{array}$ & JHACG & - Patients who underwent microscopic or & •Complications \\
\hline & & Database & & $\cdot n=746$ & $\cdot$ LOS \\
\hline & & & & & •Total hospital charges \\
\hline & & & & & -Readmission rates \\
\hline \multirow[t]{4}{*}{$\begin{array}{l}\text { Theriault et } \\
\text { al., } 2020\end{array}$} & Retrospective & $\begin{array}{l}\text { Single institution } \\
\text { database }\end{array}$ & $\mathrm{mFl}-11$ & $\begin{array}{l}\text {-Patients who underwent surgery for } \\
\text { intracranial meningioma }\end{array}$ & •LOS \\
\hline & & & & $\cdot n=76$ & •Discharge disposition \\
\hline & & & & & - Reoperation rates \\
\hline & & & & & -Readmission rates \\
\hline
\end{tabular}

Abbreviations: LOS: length of stay. NSQIP: National Surgical Quality Improvement Program. 


\begin{tabular}{|c|c|c|c|c|c|}
\hline $\begin{array}{l}\text { Authors \& } \\
\text { Year }\end{array}$ & Study Type & Data Source & $\begin{array}{l}\text { Frailty } \\
\text { instrument }\end{array}$ & Study population & Outcomes Studied \\
\hline \multirow{6}{*}{$\begin{array}{l}\text { Youngerman } \\
\text { et al., } 2018\end{array}$} & \multirow[t]{6}{*}{ Retrospective } & \multirow[t]{6}{*}{ NSQIP database } & \multirow[t]{6}{*}{$\mathrm{mFI}-11$} & \multirow{2}{*}{$\begin{array}{l}\text {-Patients who underwent surgery for brain } \\
\text { tumors } \\
\cdot n=9,149\end{array}$} & ·30-day mortality \\
\hline & & & & & $\begin{array}{l}\text {-Severe medical } \\
\text { complications }\end{array}$ \\
\hline & & & & & $\begin{array}{l}\text {-Severe neurologic } \\
\text { complications }\end{array}$ \\
\hline & & & & & -Any complication \\
\hline & & & & & -Prolonged LOS \\
\hline & & & & & -Unfavorable discharge \\
\hline
\end{tabular}

\section{Discussion}

\section{Study Rationale}

As ascertained by a wide variety of measurement tools, frailty have been demonstrated to be an important tool in preoperative patient counseling. While still a newer concept within neuro-oncology, frailty appears poised to inform clinical decision-making in patients undergoing surgery for brain tumors. To synthesize existing research and inform next steps with this work, we systematically reviewed the literature on frailty in brain tumor patients.

\section{Summary of Evidence and Key Findings}

Nearly all (20 out of 21) identified studies were retrospective, using single-institution or national databases, representing low-level evidence with potentially high risk of bias. The most investigated frailty instrument was the mFI-5, followed by the mFI-11, JHACG, and HFS. All studies reported an association between frailty and traditional postoperative outcomes, including mortality, survival, complications, LOS, charges, costs, discharge disposition, and readmissions. While statistical methodology and reporting between studies varied, we note grossly similar effect sizes in the relative impact of higher frailty scores upon each postoperative outcome across studies.

Interestingly, all included studies were published within the past five years, with the majority published in the past two years. We speculate that this recent proliferation of publications may have resulted from multiple convergent forces, including emphasis on an aging population, personalized medicine, predictive analytics, high-value care, and publication productivity metrics.

\section{Thresholds in Frailty Instruments}

A standardized method of distinguishing among different levels of frailty within each instrument was not present. The $\mathrm{mFI}-5$ and $\mathrm{mFI}-11 \mathrm{were}$ most commonly sub-divided into three categories of frailty (no frailty, low frailty, and medium-to-high-frailty), though two studies used four categories and four studies used two categories. Some studies explicitly stated that their frailty thresholds were based on previous literature, but in many cases, there was not an explanation for how particular thresholds were determined. Furthermore, while there are statistical trends distinguishing between different categories of frailty, it is challenging to determine whether these categories precisely map to discrete biological states.

Additionally, comparison of studies using the same frailty instruments is complicated by the lack of standardization in determining frailty thresholds and by differences in reporting frailty as integers or fractions. It is also difficult to compare levels of frailty across different indices. For example, is an "intermediate" score on the mFI-5 clinically similar to an "intermediate" score on the mFI-11 or HFS? While we cannot determine whether particular conventions are superior to others, we posit that future research and clinical applications would benefit from the adoption of standard nomenclature and conventions for each frailty instrument.

\section{Comparison of Frailty Instruments}

The four frailty instruments described were developed in different contexts and consequently differ in their conceptual framework. The HFS is based on Fried's 2001 study that operationalized a definition of frailty in community-dwelling older adults[1]. The authors sought to synthesize a critical mass of phenotypic components that, taken together, would distinguish the frailty phenotype from conflated concepts of disability, comorbidity, and advanced age[1]. The JHACG was developed as a risk adjustment method for payment to Medicare plans based on comprehensive diagnostic information[8]. While the JHACG has some diagnostic and thematic overlap with the other instruments, it also includes unique elements such as malnutrition, vision impairment, decubitus ulcer, incontinence, falls, and social support need. Conceptually, it may function as a hybrid instrument capturing elements of both the frailty phenotype/physical frailty and the deficit accumulation frameworks that serve as markers of accumulated comorbid conditions. The mFI-11 was introduced in Velanovich's 2013 study to measure frailty and co-morbid conditions in national databases and clinical practice[6]. The authors mapped the 70-item Canadian Study of Health and Aging Frailty Index to corresponding variables in the NSQIP database to predict morbidity and mortality in surgical specialties. Beginning in 2013, some variables were removed from the NSQIP database, 
leaving only five of the original 11 variables remaining. Subramaniam and colleagues subsequently introduced the $\mathrm{mFI} 5$, which used the remaining five factors[7]. They found that it was an equally effective predictor of mortality and postoperative complications compared to the mFI-11[7]. Both $\mathrm{mFI}$ instruments more closely align with deficit accumulation models of frailty, though the binary functional status variable may represent a composite of elements from the frailty phenotype model.

While all frailty instruments demonstrated associations with a similar set of postoperative outcomes, it is unclear to what extent the deficit accumulation models and frailty phenotype models may converge upon similar practical applications in the clinical setting. Given that the index model contains a wide range of comorbid conditions in its measurement, and that the phenotype was conceptualized around a broader physiological approach, there are likely important biological distinction between these two models[38]. However, due to heterogeneity in study populations and design, we were unable to directly compare them. Future work should directly compare multiple frailty measurement tools in brain tumor patients and investigate the clinical implications of each.

We note that the frequency with which each frailty instrument was reported may correlate with each instrument's practical ease of use. The mFI-5 contains elements that are readily identified in patient medical records and large national databases using International Classification of Diseases codes. The mFI-11 is similar, though it is longer and contains variables that are no longer reported in the NSQIP database. The JHACG can be applied to large datasets using proprietary software available for purchase. The HFS relies upon prospective assessment of patient-reported data and objective data using specialized equipment, which in general is often not available in a medical record.

\section{Related Concepts: Sarcopenia, Nutrition}

Frailty is closely related to two additional concepts - sarcopenia and nutritional status - that have recently been explored in brain tumor patients. Sarcopenia refers to a combination of reduced muscle mass, strength, and/or physical performance [39]. Two recent studies demonstrated that sarcopenia was associated with survival in patients with glioblastoma[40,41]. Two other studies demonstrated an association between Sarcopenia and survival in patients with brain metastases from melanoma, breast cancer, and lung cancer [42, 43]. There is considerable overlap between the sarcopenia and frailty, such that sarcopenic patients may frequently be frail. A similar relationship exists with nutritional status. The JHACG and HFS may partially capture nutritional status by including criteria for "weight loss" and "shrinking," respectively. Several studies have demonstrated that malnutrition is associated with survival, Stupp protocol completion, and LOS in patients with glioblastoma[44-47]. Ultimately, both sarcopenia and nutrition remain relatively understudied but important concepts within neurosurgical oncology.

\section{Incorporating Frailty into Clinical Practice}

Prior work has described multiple models in which frailty instruments could be meaningfully incorporated into clinical practice[18]. First, frailty assessment could be rapidly performed preoperatively, perhaps with the assistance of web-based calculators[19, 48], nomograms[49], or other decision support tools. Select frail patients could then undergo multisystem perioperative optimization (i.e., "prehabilitation") to improve potentially modifiable characteristics before surgery[10]. Depending on the frailty measurement tool, methods of prehabilitation may include reducing vulnerability resulting from certain disease condition or exercise-based prehabilitation process to improve sarcopenia. Postoperatively, frailty could help guide selection of level of care and follow-up with health providers[18,50]. Both types of frailty assessment maybe necessary to optimize preand post-operative care.

\section{Future Directions}

Important next steps could include investigation into an optimal conceptual framework and frailty instrument to assess frailty with expanded outcomes and adoption of standard conventions to report frailty. This work would benefit from direct comparisons of various frailty instruments on well-defined patient populations. Ideally, such studies could also include other frailty instruments that have been popularized in the surgical literature but not yet applied to brain tumor patients. All frailty studies in brain tumor patients to date have looked at traditional postoperative outcomes; however, we suspect that frailty may similarly predict patient-reported and quality of life outcomes. Finally, there is a need for high-quality, prospective studies and clinical implementation of frailty assessment to meaningfully "move the needle".

\section{Limitations}

Our systematic review was limited by the quality of the included studies. Included studies were almost all retrospective with significant heterogeneity in assessment methodology and patient populations. We were therefore unable to perform a meta-analysis. Included studies may also be influenced by selection, reporting, publication, and other biases. Furthermore, our inclusion and exclusion criteria precluded incorporation of potentially interesting studies in which frailty was discussed but not explicitly associated with postoperative outcomes. Additionally, we did not include papers that discussed frailty but only measured Karnofsky performance status, Eastern Cooperative Oncology Group performance status, Charlson Comorbidity Index, Charlson-Deyo score, body mass index, or albumin as markers of health.

We were also limited by reporting of data. Summary data for frailty scores were reported where available. In some cases, frailty scores, odds ratios, and beta coefficients were not clearly described and could not be precisely obtained from provided figures and text. They were consequently not included in our summary table. Similarly, papers that mentioned frailty but did not clearly describe the components of the frailty instrument(s) under investigation or the contribution of frailty to other scoring systems were excluded.

Page $12 / 16$ 


\section{Conclusion}

Frailty is an increasingly popular concept in brain tumor patients that is associated with important postoperative outcomes, including mortality, survival, complications, LOS, charges, costs, discharge disposition, readmissions, and operation time. However, the extant literature is comprised largely of retrospective studies using multiple frailty instruments that have different conceptual frameworks, different thresholds to define levels of frailty, and heterogeneous patient populations. Frailty ascertainment appears well-suited to inform clinical decision-making in brain tumor patients, but further work is first needed to understand best practices and tools needed to assess frailty and apply these concepts to clinical practice.

\section{Abbreviations}

LOS: Length of Stay,HFS: Hopkins Frailty Score, JHACG: Johns Hopkins Adjusted Clinical Groups, mFI-5: 5-factor modified frailty index, mFI-11: 11factor modified frailty index, NSQIP: National Surgical Quality Improvement Program, OCEBM: Oxford Center for Evidence-Based Medicine, PRISMA: Preferred Reporting Items for Systematic Reviews and Meta-Analyses

\section{Declarations}

Reporting Guidelines: Preferred Reporting Items for Systematic Reviews and Meta-Analyses (PRISMA) guidelines were followed for this study.

Disclosures:Authors have no disclosures and declare no competing interests with respect to this work.

Acknowledgements: Dr. Walston is supported by the Johns Hopkins Older Americans Independence Center, an NIA supported P-30 program that focuses on frailty in older adults. We thank the Johns Hopkins Neuro-Oncology Surgical Outcomes Laboratory for helpfulcomments and edits.

Funding: The authors declare that no funds, grants, or other support were received during the preparation of this manuscript

Competing Interests: The authors have no relevant financial or non-financial interests to disclose

Author Contributions: Sakibul Huq contributed to the study conception and design. A systematic review of the English peer-reviewed literature was conducted by Stella Seal. Articles screening and analyzing were conducted by Sakibul Huq, Jiaqi Liu and Robert Romano. The first draft of the manuscript was written by Sakibul Huq and all authors commented on previous versions of the manuscript. All authors read and approved the final manuscript

Data Availability: Not applicable

Ethics approval: Not applicable

Consent to participate: Not applicable

Consent to publish: Not applicable

\section{References}

1. Fried LP, Tangen CM, Walston J, Newman AB, Hirsch C, Gottdiener J, Seeman T, Tracy R, Kop WJ, Burke G, McBurnie MA (2001) Frailty in older adults: evidence for a phenotype. J Gerontol A Biol Sci Med Sci 56: M146-156 doi:10.1093/gerona/56.3.m146

2. (2021) Conceptual Models of Frailty. https://frailtyscience.org/conceptual-models-frailty. Accessed August 122021

3. Buchner DM, Wagner EH (1992) Preventing frail health. Clin Geriatr Med 8: 1-17

4. Mitnitski AB, Mogilner AJ, Rockwood K (2001) Accumulation of deficits as a proxy measure of aging. ScientificWorldJournal 1: $323-336$ doi:10.1100/tsw.2001.58

5. Makary MA, Segev DL, Pronovost PJ, Syin D, Bandeen-Roche K, Patel P, Takenaga R, Devgan L, Holzmueller CG, Tian J, Fried LP (2010) Frailty as a predictor of surgical outcomes in older patients. Journal of the American College of Surgeons 210: 901-908 doi:10.1016/j.jamcollsurg.2010.01.028

6. Velanovich V, Antoine H, Swartz A, Peters D, Rubinfeld I (2013) Accumulating deficits model of frailty and postoperative mortality and morbidity: its application to a national database. Journal of Surgical Research 183: 104-110 doi:https://doi.org/10.1016/j.jss.2013.01.021

7. Subramaniam S, Aalberg JJ, Soriano RP, Divino CM (2018) New 5-Factor Modified Frailty Index Using American College of Surgeons NSQIP Data. Journal of the American College of Surgeons 226: 173-181.e178 doi:10.1016/j.jamcollsurg.2017.11.005

8. Abrams C LR, Weiner JP (2003) Development and evaluation of the Johns Hopkins University risk adjustment models for Medicare+Choice plan payment. Johns Hopkins University, Baltimore, MD

9. Walston JD, Bandeen-Roche K (2015) Frailty: a tale of two concepts. BMC Med 13: 185 doi:10.1186/s12916-015-0420-6 
10. Hall DE, Arya S, Schmid KK, Blaser C, Carlson MA, Bailey TL, Purviance G, Bockman T, Lynch TG, Johanning J (2017) Development and Initial Validation of the Risk Analysis Index for Measuring Frailty in Surgical Populations. JAMA Surg 152: 175-182 doi:10.1001/jamasurg.2016.4202

11. Asemota AO, Gallia GL (2019) Impact of frailty on short-term outcomes in patients undergoing transsphenoidal pituitary surgery. Journal of neurosurgery 132: 360-370 doi:10.3171/2018.8.Jns18187510.3171/2018.8.JNS181875.

12. Bonney PA, Chartrain AG, Briggs RG, Jarvis CA, Ding L, Mack WJ, Zada G, Attenello FA (2021) Frailty Is Associated with In-Hospital Morbidity and Nonroutine Disposition in Brain Tumor Patients Undergoing Craniotomy. World neurosurgery 146: e1045-e1053 doi:10.1016/j.wneu.2020.11.083

13. Casazza GC, McIntyre MK, Gurgel RK, McCrary HC, Shelton C, Couldwell WT, Bowers CA (2020) Increasing Frailty, Not Increasing Age, Results in Increased Length of Stay Following Vestibular Schwannoma Surgery. Otol Neurotol 41: e1243-e1249 doi:10.1097/mao.000000000000283110.1097/MAO.0000000000002831.

14. Cloney M, D'Amico R, Lebovic J, Nazarian M, Zacharia BE, Sisti MB, Bruce JN, McKhann GM, Iwamoto FM, Sonabend AM (2016) Frailty in Geriatric Glioblastoma Patients: A Predictor of Operative Morbidity and Outcome. World neurosurgery 89: $362-367$ doi:10.1016/j.wneu.2015.12.096

15. Goshtasbi K, Abouzari M, Soltanzadeh-Zarandi S, Sarna B, Lee A, Hsu FPK, Djalilian HR (2020) The association of age, body mass index, and frailty with vestibular schwannoma surgical morbidity. Clinical Neurology and Neurosurgery 197 doi:10.1016/j.clineuro.2020.106192

16. Harland TA, Wang M, Gunaydin D, Fringuello A, Freeman J, Hosokawa PW, Ormond DR (2020) Frailty as a Predictor of Neurosurgical Outcomes in Brain Tumor Patients. World neurosurgery 133: e813-e818 doi:10.1016/j.wneu.2019.10.010

17. Henry RK, Reeves RA, Wackym PA, Ahmed OH, Hanft SJ, Kwong KM (2021) Frailty as a Predictor of Postoperative Complications Following Skull Base Surgery. The Laryngoscope doi:10.1002/lary.2948510.1002/lary.29485.

18. Huq S, Khalafallah AM, Jimenez AE, Gami A, Lam S, Ruiz-Cardozo MA, Oliveira LAP, Mukherjee D (2020) Predicting Postoperative Outcomes in Brain Tumor Patients With a 5-Factor Modified Frailty Index. Neurosurgery doi:10.1093/neuros/nyaa335

19. Huq S, Khalafallah AM, Patel P, Sharma P, Dux H, White T, Jimenez AE, Mukherjee D (2021) Predictive Model and Online Calculator for Discharge Disposition in Brain Tumor Patients. World neurosurgery 146: e786-e798 doi:10.1016/j.wneu.2020.11.018

20. Jimenez AE, Khalafallah AM, Huq S, Horowitz MA, Azmeh O, Lam S, Oliveira LAP, Brem H, Mukherjee D (2020) Predictors of Nonroutine Discharge Disposition Among Patients with Parasagittal/Parafalcine Meningioma. World neurosurgery 142: e344-e349 doi:10.1016/j.wneu.2020.06.23910.1016/j.wneu.2020.06.239. Epub 2020 Jul 9.

21. Khalafallah AM, Shah PP, Huq S, Jimenez AE, Patel PP, London NR, Hamrahian AH, Salvatori R, Gallia GL, Rowan NR, Mukherjee D (2020) The 5factor modified frailty index predicts health burden following surgery for pituitary adenomas. Pituitary doi:10.1007/s11102-020-01069-5

22. Khalafallah AM, Huq S, Jimenez AE, Brem H, Mukherjee D (2020) The 5-factor modified frailty index: an effective predictor of mortality in brain tumor patients. Journal of neurosurgery doi:10.3171/2020.5.Jns20766

23. Khalafallah AM, Jimenez AE, Patel P, Huq S, Azmeh O, Mukherjee D (2020) A novel online calculator predicting short-term postoperative outcomes in patients with metastatic brain tumors. Journal of neuro-oncology doi:10.1007/s11060-020-03626-1

24. Katiyar V, Sharma R, Tandon V, Goda R, Ganeshkumar A, Suri A, Chandra PS, Kale SS (2020) Impact of frailty on surgery for glioblastoma: a critical evaluation of patient outcomes and caregivers' perceptions in a developing country. Neurosurgical focus 49: E14 doi:10.3171/2020.7.Focus2048210.3171/2020.7.FOCUS20482.

25. Richardson AM, McCarthy DJ, Sandhu J, Mayrand R, Guerrero C, Rosenberg C, Gernsback JE, Komotar R, Ivan M (2019) Predictors of Successful Discharge of Patients on Postoperative Day 1 After Craniotomy for Brain Tumor. World neurosurgery 126: e869-e877 doi:10.1016/j.wneu.2019.03.00410.1016/j.wneu.2019.03.004. Epub 2019 Mar 9.

26. Sastry RA, Pertsch NJ, Tang O, Shao B, Toms SA, Weil RJ (2020) Frailty and outcomes after craniotomy for brain tumor. J Clin Neurosci 81: 95100 doi:10.1016/j.jocn.2020.09.00210.1016/j.jocn.2020.09.002. Epub 2020 Oct 2.

27. Schneider M, Potthoff AL, Scharnböck E, Heimann M, Schäfer N, Weller J, Schaub C, Jacobs AH, Güresir E, Herrlinger U, Vatter H, Schuss P (2020) Newly diagnosed glioblastoma in geriatric (65+) patients: impact of patients frailty, comorbidity burden and obesity on overall survival. Journal of neuro-oncology 149: 421-427 doi:10.1007/s11060-020-03625-210.1007/s11060-020-03625-2. Epub 2020 Sep 29.

28. Shahrestani S, Ballatori AM, Chen XT, Ton A, Strickland BA, Brunswick A, Zada G (2020) Analysis of modifiable and nonmodifiable risk factors in patients undergoing pituitary surgery. Journal of neurosurgery: 1-8 doi:10.3171/2020.4.Jns2041710.3171/2020.4.JNS20417.

29. Shahrestani S, Lehrich BM, Tafreshi AR, Brown NJ, Lien BV, Ransom S, Ransom RC, Ballatori AM, Ton A, Chen XT, Sahyouni R (2020) The Role of Frailty in Geriatric Cranial Neurosurgery for Primary Central Nervous System Neoplasms. Neurosurgical focus 49: 1-8 doi:10.3171/2020.7.FOCUS20426

30. Theriault BC, Pazniokas J, Adkoli AS, Cho EK, Rao N, Schmidt M, Cole C, Gandhi C, Couldwell WT, Al-Mufti F, Bowers CA (2020) Frailty predicts worse outcomes after intracranial meningioma surgery irrespective of existing prognostic factors. Neurosurgical focus 49 : E16 doi:10.3171/2020.7.Focus2032410.3171/2020.7.FOCUS20324.

31. Youngerman BE, Neugut Al, Yang J, Hershman DL, Wright JD, Bruce JN (2018) The modified frailty index and 30-day adverse events in oncologic neurosurgery. Journal of neuro-oncology 136: 197-206 doi:10.1007/s11060-017-2644-0

Page $14 / 16$ 
32. Pazniokas J, Gandhi C, Theriault B, Schmidt M, Cole C, Al-Mufti F, Santarelli J, Bowers CA (2021) The immense heterogeneity of frailty in neurosurgery: a systematic literature review. Neurosurg Rev 44: 189-201 doi:10.1007/s10143-020-01241-2

33. Page MJ, McKenzie JE, Bossuyt PM, Boutron I, Hoffmann TC, Mulrow CD, Shamseer L, Tetzlaff JM, AkI EA, Brennan SE, Chou R, Glanville J, Grimshaw JM, Hróbjartsson A, Lalu MM, Li T, Loder EW, Mayo-Wilson E, McDonald S, McGuinness LA, Stewart LA, Thomas J, Tricco AC, Welch VA, Whiting P, Moher D (2021) The PRISMA 2020 statement: an updated guideline for reporting systematic reviews. BMJ 372 : n71 doi:10.1136/bmj.n71

34. OCEBM OCEBM Levels of Evidence. https://www.cebm.net/index.aspx?o=5653.

35. Institute OHR (2021) The Newcastle-Ottawa Scale (NOS) for assessing the quality of nonrandomised studies in meta-analyses. http://www.ohri.ca/programs/clinical_epidemiology/oxford.asp.

36. Gomes dos Santos A, de Carvalho RF, de Morais ANLR, Silva TM, Baylão VMR, Azevedo M, de Oliveira AJM (2021) Role of neutrophillymphocyte ratio as a predictive factor of glioma tumor grade: A systematic review. Crit Rev Oncol Hematol 163: 103372 doi:https://doi.org/10.1016/j.critrevonc.2021.103372

37. He L, Zhang B, Zhang J, Guo Z, Shi F, Zeng Q (2021) Effectiveness of Postoperative Adjuvant Radiotherapy in Atypical Meningioma Patients After Gross Total Resection: A Meta-Analysis Study. Frontiers in oncology 10: 556575-556575 doi:10.3389/fonc.2020.556575

38. Fried LP, Cohen AA, Xue Q-L, Walston J, Bandeen-Roche K, Varadhan R (2021) The physical frailty syndrome as a transition from homeostatic symphony to cacophony. Nature Aging 1: 36-46 doi:10.1038/s43587-020-00017-z

39. Dunne RF, Loh KP, Williams GR, Jatoi A, Mustian KM, Mohile SG (2019) Cachexia and Sarcopenia in Older Adults with Cancer: A Comprehensive Review. Cancers (Basel) 11 doi:10.3390/cancers11121861

40. Furtner J, Genbrugge E, Gorlia T, Bendszus M, Nowosielski M, Golfinopoulos V, Weller M, van den Bent MJ, Wick W, Preusser M (2019) Temporal muscle thickness is an independent prognostic marker in patients with progressive glioblastoma: translational imaging analysis of the EORTC 26101 trial. Neuro-Oncology 21: 1587-1594 doi:10.1093/neuonc/noz131

41. Huq S, Khalafallah AM, Ruiz-Cardozo MA, Botros D, Oliveira LAP, Dux H, White T, Jimenez AE, Gujar SK, Sair HI, Pillai JJ, Mukherjee D (2021) A novel radiographic marker of sarcopenia with prognostic value in glioblastoma. Clin Neurol Neurosurg 207: 106782 doi:10.1016/j.clineuro.2021.106782

42. Furtner J, Berghoff AS, Albtoush OM, Woitek R, Asenbaum U, Prayer D, Widhalm G, Gatterbauer B, Dieckmann K, Birner P, Aretin B, Bartsch R, Zielinski CC, Schöpf V, Preusser M (2017) Survival prediction using temporal muscle thickness measurements on cranial magnetic resonance images in patients with newly diagnosed brain metastases. European Radiology 27: 3167-3173 doi:10.1007/s00330-016-4707-6

43. Furtner J, Berghoff AS, Schöpf V, Reumann R, Pascher B, Woitek R, Asenbaum U, Pelster S, Leitner J, Widhalm G, Gatterbauer B, Dieckmann K, Höller C, Prayer D, Preusser M (2018) Temporal muscle thickness is an independent prognostic marker in melanoma patients with newly diagnosed brain metastases. Journal of neuro-oncology 140: 173-178 doi:10.1007/s11060-018-2948-8

44. Han S, Huang Y, Li Z, Hou H, Wu A (2015) The prognostic role of preoperative serum albumin levels in glioblastoma patients. BMC Cancer 15: 108-108 doi:10.1186/s12885-015-1125-0

45. Xu WZ, Li F, Xu ZK, Chen X, Sun B, Cao JW, Liu YG (2017) Preoperative albumin-to-globulin ratio and prognostic nutrition index predict prognosis for glioblastoma. Onco Targets Ther 10: 725-733 doi:10.2147/ott.S127441

46. Rigamonti A, Imbesi F, Silvani A, Lamperti E, Agostoni E, Porcu L, De Simone I, Torri V, Ciusani E, Bonato C, Salmaggi A (2019) Prognostic nutritional index as a prognostic marker in glioblastoma: Data from a cohort of 282 Italian patients. J Neurol Sci 400: 175-179 doi:10.1016/j.jns.2019.04.002

47. Huq S, Khalafallah AM, Botros D, Oliveira LAP, White T, Dux H, Jimenez AE, Mukherjee D (2021) The Prognostic Impact of Nutritional Status on Postoperative Outcomes in Glioblastoma. World neurosurgery 146: e865-e875 doi:10.1016/j.wneu.2020.11.03310.1016/j.wneu.2020.11.033. Epub 2020 Nov 13.

48. Khalafallah AM, Jimenez AE, Patel P, Huq S, Azmeh O, Mukherjee D (2020) A novel online calculator predicting short-term postoperative outcomes in patients with metastatic brain tumors. Journal of neuro-oncology 149: 429-436 doi:10.1007/s11060-020-03626-1

49. Lubelski D, Ehresman J, Feghali J, Tanenbaum J, Bydon A, Theodore N, Witham T, Sciubba DM (2020) Prediction calculator for nonroutine discharge and length of stay after spine surgery. The Spine Journal doi:https://doi.org/10.1016/j.spinee.2020.02.022

50. Laan MT, Roelofs S, Van Huet I, Adang EMM, Bartels R (2020) Selective Intensive Care Unit Admission After Adult Supratentorial Tumor Craniotomy: Complications, Length of Stay, and Costs. Neurosurgery 86: E54-e59 doi:10.1093/neuros/nyz388

\section{Figures}


Identification of studies via databases and registers

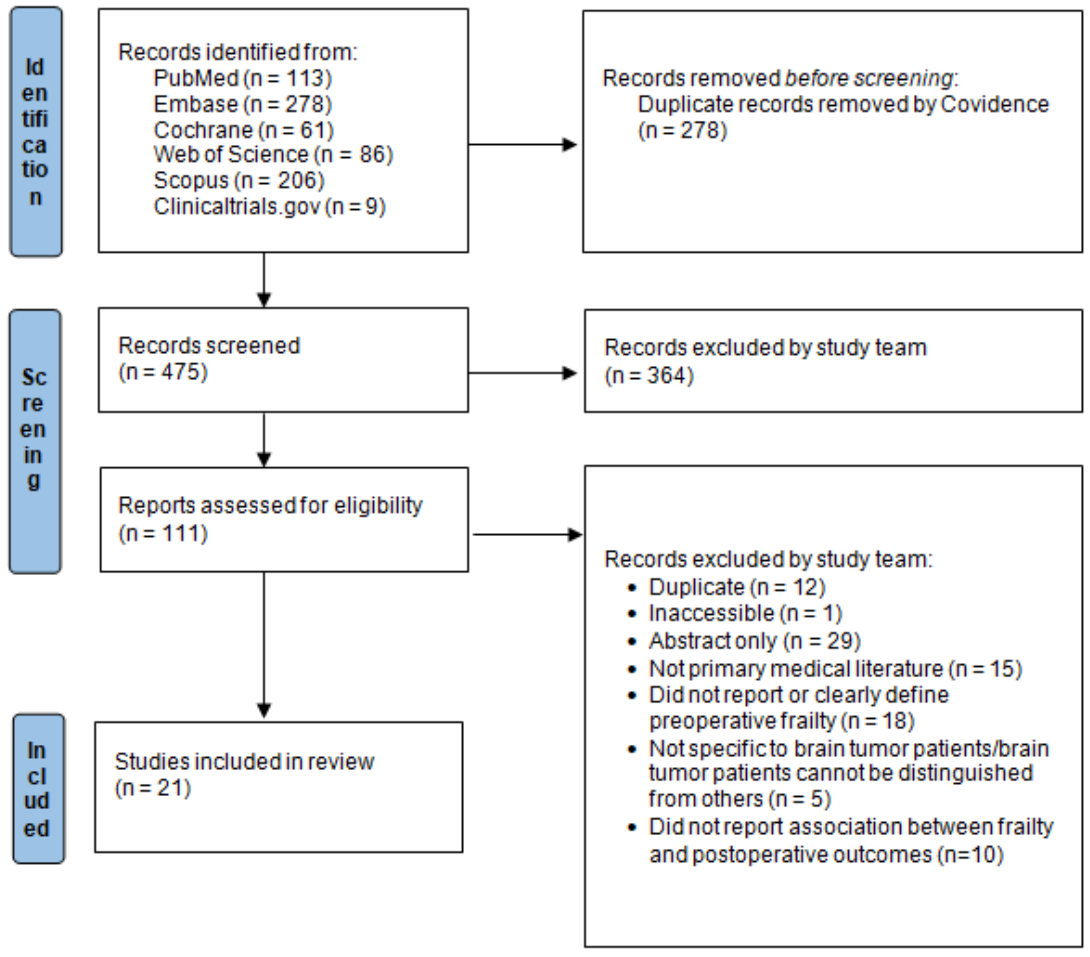

Figure 1

Preferred Reporting Items for Systematic Reviews and Meta-Analyses (PRISMA) Flow Diagram. From: Page MJ, McKenzie JE, Bossuyt PM, Boutron I, Hoffmann TC, Mulrow CD, et al. The PRISMA 2020 statement: an updated guideline for reporting systematic reviews. BMJ 2021;372:n71. doi: 10.1136/bmj.n71. 